\title{
Systematic Review of Premenstrual, Postmenstrual and Infertility Disorders of Vitex Agnus Castus
}

\author{
Mahmoud Rafieian-Kopaei ${ }^{1}$, Mino Movahedi²
}

${ }^{1}$ Ph.D. in Pharmacology, Full Professor, Medical Plants Research Center, Shahrekord University of Medical Sciences, Shahrekord, Iran

${ }^{2}$ M.D., Gynecologist, Assistant Professor, Faculty of Medicine, Isfahan University of Medical Sciences, Isfahan, Iran

Type of article: Systematic review

\begin{abstract}
Introduction: Vitex agnus-castus, also called vitex is aboriginal to the Mediterranean region, with long leaves, tender stem, flowers and ripening seeds. The aim of this study was to overview premenstrual, postmenstrual and infertility disorder of Vitex agnus-castus.

Methods: This review article was carried out by searching studies in PubMed, Medline, Web of Science, and IranMedex databases. The initial search strategy identified about 87 references. In this study, 43 studies were accepted for further screening, and met all our inclusion criteria (in English, full text, therapeutic effects of Vitex agnus-castus and dated mainly from the year 2009 to 2016).

The search terms were Vitex agnus-castus, premenstrual, postmenstrual, infertility disorder properties and pharmacological effects.

Result: Vitex agnus-castus was shown to contribute to the treatment of premenstrual syndrome (PMS). Moreover, the result of the present study showed that this valuable plant is helpful in alleviation of pain resulting from postmenstrual disease. Furthermore, it was found that Vitex agnus-castus is beneficial in infertility disorder.

Conclusion: Vitex agnus-castus (AC) is a phytopharmaceutical compound and is shown to be widely used to treat PMS and PMDD. In addition, it was shown to be beneficial in post-menstrual cases and it can also contribute to treatment of infertility cases in both men and women. Dopaminergic compounds available in this plant help to treat premenstrual mastodynia as well as other symptoms of the premenstrual syndrome.

Keywords: Vitex agnus-castusis, Phytochemicals, Therapeutic effects, Pharmacognosy, Alternative medicine

\section{Introduction}

\subsection{Background}

Botanical medicine has been traditionally used for medicinal purposes to cure many kinds of morbidity (1-13). Vitex agnus-castus, also called vitex is aboriginal to the Mediterranean region with long leaves and tender stem, along with flowers and ripening seeds. The main Chemical compound of this plant contain vitexin, casticin, agnuside, $\mathrm{p}$ hydroxybenzoic acid, alkaloids, diterpenoids. Its fruit contain flavonoids, terpenoids, neolignans, and phenolic compounds, as well as one glyceride. The plant contributes to improve many morbidities including premenstrual syndrome, mastalgia, inflammation and sexual dysfunction, and also helps to relieve pain, and possesses antinociceptive effects (14). Vitex agnus-castus is effective in reducing symptoms of many gynecological problems. It is clinically used in abnormal uterine bleeding disorders and mastodynia. This plant is useful in mild hyperprolactinemia and luteal phase defect. It is also helpful in alleviating menstruation, bleeding management uterine fibroids, polycystic ovarian syndrome, prostate disorders, migrainous women with premenstrual syndrome.
\end{abstract}

\section{Corresponding author:}

Assistant Professor Dr. Mino Movahedi, Faculty of Medicine, Isfahan University of Medical Sciences, Isfahan, Iran. Tel: +98.9131192140, Fax: +98.32225456, Email: saeidi@med.mui.ac.ir

Received: September 24, 2016, Accepted: November 30, 2016, Published: January 2017

iThenticate screening: December 18, 2016, English editing: January 08, 2017, Quality control: January 17, 2017

(C) 2017 The Authors. This is an open access article under the terms of the Creative Commons Attribution-NonCommercialNoDerivs License, which permits use and distribution in any medium, provided the original work is properly cited, the use is non-commercial and no modifications or adaptations are made. 


\subsection{Statement of problem}

Herbalism, the use of plants for medical treatment, is still widely practiced today. The evidence from clinical trial studies show that herbalism could fulfill the prerequisite of humankind to cure their diseases. Organic, cost effective, compatible with the body and having less adverse effects, causes people to be more willing to partake of herbs for medicinal purposes, but there is still perplexity about their effectiveness, dosage, toxicity as well as standardization. Thus, a lot of research has been conducted to explore botanical plants generating useful chemical and pharmacological structure.

\subsection{Objective of research}

This review article is aimed to overview premenstrual, postmenstrual and infertility disorders of Vitex agnus-castus.

\section{Material and Methods}

\subsection{Searching databases}

This review article was carried out by searching studies in PubMed, Medline, Web of Science, and IranMedex databases. The initial search strategy identified about 87 references. In this study, 43 studies were accepted for further screening and met all our inclusion criteria (in English, full text, therapeutic effects of Vitex agnus-castus L and dated mainly from the year 1997 to 2016). The search terms were: Vitex agnus-castus, premenstrual, postmenstrual, infertility disorder properties, and pharmacological effects.

\subsection{Inclusion and exclusion criteria}

Inclusion criteria were the following key words which were used to search for the relevant articles published from 1997 to 2016 whose full text should be available in English. Articles included consisted of clinical trials, in vitro, in vivo, review, or meta-analysis studies. Exclusion criteria were relating to other properties of this herb than its antipremenstrual, anti-postmenstrual and anti-infertility activities, only abstract was available, not in the time line of study. Those articles that did not match our inclusion criteria (in languages other than English, between the time lines of study) were excluded from the study.

\subsection{Quality assessment}

The inclusion and exclusion criteria were checked. Based on our search strategy, 54 articles were included; meanwhile, 11 studies were excluded from the study; 43 articles were left for more study.

\section{Results}

\subsection{Pre-menstrual stress syndrome (PMS)}

The effect of Vitex agnus-castus extract in women with premenstrual syndrome (PMS) was investigated. The plant showed to be effective, safe and well tolerated (15). The VAC extract was shown to be efficient in the treatment of hyperprolactinemia, menstrual disorders, PMS and mastalgia. The VAC benefits are its high tolerability, with no side-effects and no drug interactions (16). In a study in Germany, the cases of PMS/PMDD were investigated in which the plant extract Vitex agnus-castus was used as a complementary method to heal PMS/PMDD (17). In another study, patients with PMDD were treated with fluoxetine and Vitex agnus-castus. The fluoxetine helped to treat psychological symptoms while the VAC extract reduced the physical symptoms (18). Efficacy of Vitex agnuscastus extract in premenstrual syndrome was assessed in China. Results showed that the plant is very beneficial in moderate-to-severe PMS treatment (19). The plant extract BNO 1095 shows powerful effects in treating moderate to serious PMS, particularly in manifestations of negative effect and water maintenance (20). Additionally, Vitex agnus can relieve mild and moderate PMS symptoms (21). In another study, it was found that Vitex agnus-castus extract Ze 440 is helpful in PMS improvement in the case of gradual return after cessation (22). The VAC extract Ze 440 can relieve PMS symptoms in a dose of $20 \mathrm{mg}$ (23). In comparison with pyridoxine, VAC markedly alleviates PMTS complaints, including bosom delicacy, edema, inward pressure, cerebral pain, obstruction, and depression. Thus, Agnolyt ${ }^{\circledR}$ capsules were shown to be helpful in the treatment of PMTS (24). The new Vitex preparation showed high efficiency, and is safe for all aspects of PMS, compared to other Vitex preparations (25). In a Japanese population, VAC extract improved PMS symptoms with no marked side effects (26). The use of VAC in migrainous women with PMS proved to be safe and well tolerated, and decreased migraine attack frequency and duration (27). Vitex agnus-castus fruit extract decreases pathophysiological increased prolactin serum levels and could be an effective treatment for women suffering from premenstrual syndrome (28). These results suggest VAC may exert its therapeutic effects through the activation of MOR, DOR, but not KOR (29). VAC acted as an agonist at the muopiate receptor (30). Combination of Hypericum perforatum and Vitex agnus-castus are useful in PMS treatment 
among premenopausal women. However, further research is required to warrant its effect (31). In a clinical trial study, intensity of breast pain diminished quicker with VACS group. The tolerability was satisfactory, and VACS was found to be useful in the treatment of cyclical breast pain in women (32).

\subsection{Postmenopausal period}

The crude extract and butanolic fraction of VAC from Vitex agnus-castus demonstrated to be effective in prohibiting frequent causes of abnormal liver functions in the postmenopausal period (33). Vitex agnus-castus alleviates menopausal symptoms suggesting that more research may be appropriate (34). It protects the cortical and additionally the trabecular bone and may be a sheltered option for HRT. Testosterone supplementation affects trabecular bone, which are all the while balanced by the loss of cortical bone (35).

\subsection{Anti-Fertility disorders}

The result of a clinical trial study confirmed anti-fertility effects of Vitex agnus-castus L. (36). Linoleic acid present in the fruits of the plant possess the ability to bind to estrogen receptors and cause some estrogen genes induction (37). Estrogenic activities are intervened by means of estrogen receptors (ER). The flavonoid apigenin was disconnected and distinguished as the most dynamic ERss-particularly phytoestrogen in VAC. Other disconnected mixes were vitexin and penduletin. The outcome shows that the phytoestrogens in VAC are ERss-specific (38). In a male study, it was demonstrated that dopamine controls the gonadotroph-leydig cells hub. It creates the impression that VAC applies impacts through dopaminergic framework and different pathways. The result of this research demonstrates that VAC concentrate can be utilized for obsessive instances of expanding LH and testosterone (39).

\section{Discussion and conclusions}

PMS and its derivative PMDD are medical disorders (40). PMS possesses a variety of symptoms including anxiety moodiness, melancholia, breasts sensitivity and food desire (15). The premenstrual syndrome is associated with hormonal and neuronal dysbalance, diet and lifestyle. Hyperprolactinaemia is an important cause considered to be part of the endocrine disorder. PMDD is one of the major complications of the premenstrual phase (41). Vitex agnus-castus is shown to be widely used to treat PMS and PMDD. In addition, it was shown to be beneficial in postmenstrual cases and it also contributes in anti-infertility treatment. Dopaminergic compounds available in Vitex agnus-castus contribute to improve premenstrual mastodynia as well as other symptoms of the premenstrual syndrome. Some of the chemical compounds present in the plant influence the pituitary gland explaining its effects on hormonal levels. A decrease of prolactin will affect the levels of follicle-stimulating hormones (FSH) and estrogen in women and testosterone in men. In conclusion, Vitex agnus-castus (AC) is a phytopharmaceutical compound shown to be widely used to treat PMS and PMDD. Furthermore, it was shown to be beneficial in postmenstrual cases. In addition, it contributes to treatment of infertility cases in both men and women. Dopaminergic compounds found in the plant are helpful in improving premenstrual mastodynia as well as other symptoms of the premenstrual syndrome.

\section{Acknowledgments:}

The author of this manuscript would like to thank the Research and Technology Deputy of Shahrekord University of Medical Sciences for their technical and financial support. Moreover, we appreciate all those whom cooperated in this research.

\section{Conflict of Interest:}

There is no conflict of interest to be declared.

\section{Authors' contributions:}

Both authors contributed to this project and article equally. Both authors read and approved the final manuscript.

\section{References:}

1) Nasri H, Hajian S, Ahmadi A, Baradaran A, Kohi G, Nasri P, et al. Ameliorative effect of green tea against contrast-induced renal tubular cell injury. Iran J Kidney Dis. 2015; 9(6): 421-6. PMID: 26552347.

2) Bahmani M, Sarrafchi A, Shirzad H, Rafieian-Kopaei M. Autism: Pathophysiology and Promising Herbal Remedies. Curr Pharm Des. 2016; 22(3): 277-85. doi: 10.2174/1381612822666151112151529. PMID: 26561063.

3) Nasri H, Rafieian-Kopaei M. Comment on: a The effects of green tea consumption on metabolic and anthropometric indices in patients with type 2 diabetes. J Res Med Sci. 2014; 19(12): 1203-4. PMID: 25709665, PMCID: PMC4333532. 
4) Nasri H, Ahmadi A, Baradaran A, Nasri P, Hajian S, Pour-Arian A, et al. A biochemical study on ameliorative effect of green tea (Camellia sinensis) extract against contrast media induced acute kidney injury. J Renal Inj Prev. 2014; 3(2): 47-9. doi: 10.12861/jrip.2014.16. PMID: 25340167, PMCID: PMC4206045.

5) Rafieian-Kopaei M, Saki K, Bahmani M, Ghafourian S, Sadeghifard N, Taherikalani M. Listeriosis Phytotherapy: A Review Study on the Effectiveness of Iranian Medicinal Plants in Treatment of Listeriosis. J Evid Based Complementary Altern Med. 2015. doi: 10.1177/2156587215621460. PMID: 26682768.

6) Shaygannia E, Bahmani M, Zamanzad B, Rafieian-Kopaei M. A Review Study on Punica granatum L. J Evid Based Complementary Altern Med. 2016; 21(3): 221-7. doi: 10.1177/2156587215598039. PMID: 26232244.

7) Miraj S, Rafieian-Kopaei, Kiani S. Melissa officinalis L: A Review Study With an Antioxidant Prospective. J Evid Based Complementary Altern Med. 2016. doi: 10.1177/2156587216663433. PMID: 27620926.

8) Baharvand-Ahmadi B, Bahmani M, Tajeddini P, Rafieian-Kopaei M, Naghdi N. An ethnobotanical study of medicinal plants administered for the treatment of hypertension. J Renal Inj Prev. 2016; 5(3): 123-8. doi: 10.15171/jrip.2016.26. PMID: 27689107, PMCID: PMC5039997.

9) Mesripour A, Rafieian-Kopaei M, Bahrami B. The effects of Anethum graveolens essence on scopolamineinduced memory impairment in mice. Res Pharm Sci. 2016; 11(2): 145-51. PMID: 27168754, PMCID: PMC4852659.

10) Asgharzade S, Rafieian-Kopaei M, Mirzaeian A, Reiisi S, Salimzadeh L. Aloe vera toxic effects: expression of inducible nitric oxide synthase (iNOS) in testis of Wistar rat. Iran J Basic Med Sci. 2015; 18(10): 967-73. PMID: 26730330, PMCID: PMC4686580.

11) Sarrafchi A, Bahmani M, Shirzad H, Rafieian-Kopaei M. Oxidative stress and Parkinson's disease: New hopes in treatment with herbal antioxidants. Curr Pharm Des. 2016; 22(2): 238-46. doi: 10.2174/1381612822666151112151653. PMID: 26561062.

12) Rouhi-Boroujeni H, Rouhi-Boroujeni H, Gharipour M, Mohammadizadeh F, Ahmadi S, Rafieian-Kopaei M. Systematic review on safety and drug interaction of herbal therapy in hyperlipidemia: a guide for internist. Acta Biomed. 2015; 86(2): 130-6. PMID: 26422426.

13) Nasri H, Ardalan MR, Rafieian-Kopaei M. Mechanistic Impacts of Medicinal Plants in Diabetic Kidney Disease. Iran J Public Health. 2014; 43(9): 1311-3. PMID: 26175992, PMCID: PMC4500440.

14) Khalilzadeh E, Vafaei Saiah G, Hasannejad H, Ghaderi A, Ghaderi S, Hamidian G, et al. Antinociceptive effects, acute toxicity and chemical composition of Vitex agnus-castus essential oil. Avicenna J Phytomed. 2015; 5(3): 218-30. PMID: 26101755, PMCID: PMC4469960.

15) He Z, Chen R, Zhou Y, Geng L, Zhang Z, Chen S, et al. Treatment for premenstrual syndrome with Vitex agnus castus: A prospective, randomized, multi-center placebo controlled study in China. Maturitas. 2009; 63(1): 99-103. doi: 10.1016/j.maturitas.2009.01.006. PMID: 19269753.

16) Jang SH, Kim DI, Choi MS. Effects and treatment methods of acupuncture and herbal medicine for premenstrual syndrome/premenstrual dysphoric disorder: systematic review. BMC Complement Altern Med. 2014; 14: 11. doi: 10.1186/1472-6882-14-11. PMID: 24410911, PMCID: PMC3898234.

17) Weisz G, Knaapen L. Diagnosing and treating premenstrual syndrome in five western nations. Soc Sci Med. 2009; 68(8): 1498-505. doi: 10.1016/j.socscimed.2009.01.036. PMID: 19233531.

18) Atmaca M, Kumru S, Tezcan E. Fluoxetine versus Vitex agnus castus extract in the treatment of premenstrual dysphoric disorder. Hum Psychopharmacol. 2003; 18(3): 191-5. doi: 10.1002/hup.470. PMID: 12672170.

19) Ma L, Lin S, Chen R, Zhang Y, Chen F, Wang X. Evaluating therapeutic effect in symptoms of moderate - to - severe premenstrual syndrome with Vitex agnus castus (BNO 1095) in Chinese women. Aust N Z J Obstet Gynaecol. 2010; 50(2): 189-93. doi: 10.1111/j.1479-828X.2010.01137.x. PMID: 20522079.

20) Ma L, Lin S, Chen R, Wang X. Treatment of moderate to severe premenstrual syndrome with Vitex agnus castus (BNO 1095) in Chinese women. Gynecol Endocrinol. 2010; 26(8): 612-6. doi: 10.3109/09513591003632126. PMID: 20334585.

21) Zamani M, Neghab N, Torabian $S$. Therapeutic effect of Vitex agnus castus in patients with premenstrual syndrome. Acta Med Iran. 2012; 50(2): 101-6. PMID: 22359078.

22) Berger D, Schaffner W, Schrader E, Meier B, Brattström A. Efficacy of Vitex agnus castus L. extract Ze 440 in patients with pre-menstrual syndrome (PMS). Arch Gynecol Obstet. 2000; 264(3): 150-3. doi: 10.1007/s004040000123. PMID: 11129515. 
23) Schellenberg R, Zimmermann C, Drewe J, Hoexter G, Zahner C. Dose-dependent efficacy of the Vitex agnus castus extract $\mathrm{Ze} 440$ in patients suffering from premenstrual syndrome. Phytomedicine. 2012; 19(14): 1325-31. doi: 10.1016/j.phymed.2012.08.006. PMID: 23022391.

24) Lauritzen C, Reuter HD, Repges R, Böhnert KJ, Schmidt U. Treatment of premenstrual tension syndrome with Vitex agnus castus controlled, double-blind study versus pyridoxine. Phytomedicine. 1997; 4(3): 1839. doi: 10.1016/S0944-7113(97)80066-9. PMID: 23195474.

25) Loch EG, Selle H, Boblitz N. Treatment of premenstrual syndrome with a phytopharmaceutical formulation containing Vitex agnus castus. J Womens Health Gend Based Med. 2000; 9(3): 315-20. doi: 10.1089/152460900318515. PMID: 10787228.

26) Momoeda M, Sasaki H, Tagashira E, Ogishima M, Takano Y, Ochiai K. Efficacy and safety of Vitex agnus-castus extract for treatment of premenstrual syndrome in Japanese patients: a prospective, open-label study. Adv Ther. 2014; 31(3): 362-73. doi: 10.1007/s12325-014-0106-z. PMID: 24604699.

27) Ambrosini A, Di Lorenzo C, Coppola G, Pierelli F. Use of Vitex agnus-castus in migrainous women with premenstrual syndrome: an open-label clinical observation. Acta Neurol Psychiatr Belg. 2013; 113(1): 259. doi: 10.1007/s13760-012-0111-4. PMID: 22791378.

28) Döll M. [The premenstrual syndrome: effectiveness of Vitex agnus castus]. Med Monatsschr Pharm. 2009; 32(5): 186-91. PMID: 19469189.

29) Webster DE, Lu J, Chen SN, Farnsworth NR, Wang ZJ. Activation of the $\mu$-opiate receptor by Vitex agnuscastus methanol extracts: Implication for its use in PMS. J Ethnopharmacol. 2006; 106(2): 216-21. doi: 10.1016/j.jep.2005.12.025. PMID: 16439081.

30) Webster DE, He Y, Chen SN, Pauli GF, Farnsworth NR, Wang ZJ. Opioidergic mechanisms underlying the actions of Vitex agnus-castus L. Biochem pharmacol. 2011; 81(1): 170-7. doi: 10.1016/j.bcp.2010.09.013. PMID: 20854795, PMCID: PMC2993511.

31) van Die MD, Bone KM, Burger HG, Reece JE, Teede HJ. Effects of a combination of Hypericum perforatum and Vitex agnus-castus on PMS-like symptoms in late-perimenopausal women: findings from a subpopulation analysis. J Altern Complement Med. 2009; 15(9): 1045-8. doi: 10.1089/acm.2008.0539. PMID: 19757982.

32) Halaska M, Raus K, Bĕles P, Martan A, Paithner KG. [Treatment of cyclical mastodynia using an extract of Vitex agnus castus: results of a double-blind comparison with a placebo]. Ceska Gynekol. 1998; 63(5): 388-92. PMID: 9818496.

33) Moreno FN, Campos-Shimada LB, da Costa SC, Garcia RF, Cecchini AL, Natali MR, et al. Vitex agnuscastus L.(Verbenaceae) improves the liver lipid metabolism and redox state of ovariectomized rats. Evid Based Complementary Altern Med. 2015; 2015: 212378. doi: 10.1155/2015/212378. PMID: 25954315, PMCID: PMC4411462.

34) van Die MD, Burger HG, Teede HJ, Bone KM. Vitex agnus-castus (Chaste-Tree/Berry) in the treatment of menopause-related complaints. J Altern Complement Med. 2009; 15(8): 853-62. doi: 10.1089/acm.2008.0447. PMID: 19678775.

35) Sehmisch S, Boeckhoff J, Wille J, Seidlova - Wuttke D, Rack T, Tezval M, et al. Vitex agnus castus as prophylaxis for osteopenia after orchidectomy in rats compared with estradiol and testosterone supplementation. Phytother Res. 2009; 23(6): 851-8. doi: 10.1002/ptr.2711. PMID: 19107741.

36) Gorkow C, Wuttke W, März RW. [Effectiveness of Vitex agnus-castus preparations]. Wien Med Wochenschr. 2001; 152(15-16): 364-72. PMID: 12244880.

37) Liu J, Burdette JE, Sun Y, Deng S, Schlecht SM, Zheng W, et al. Isolation of linoleic acid as an estrogenic compound from the fruits of Vitex agnus-castus L.(chaste-berry). Phytomed. 2004; 11(1): 18-23. doi: 10.1078/0944-7113-00331. PMID: 14974442.

38) Jarry H, Spengler B, Porzel A, Schmidt J, Wuttke W, Christoffel V. Evidence for estrogen receptor $\beta$ selective activity of Vitex agnus-castus and isolated flavones. Planta med. 2003; 69(10): 945-7. doi: 10.1055/s-2003-45105. PMID: 14648399.

39) Nasri S, Oryan S, Rohani AH, Amin GR. The effects of Vitex agnus castus extract and its interaction with dopaminergic system on LH and testosterone in male mice. Pak J Biol Sci. 2007; 10(14): 2300-7. doi: 10.3923/pjbs.2007.2300.2307. PMID: 19070148.

40) Ciotta L, Pagano I, Stracquadanio M, Di Leo S, Ando A, Formuso C. [Psychic aspects of the premenstrual dysphoric disorders. New therapeutic strategies: our experience with Vitex agnus castus]. Minerva ginecol. 2011; 63(3): 237-45. PMID: 21654609. 